
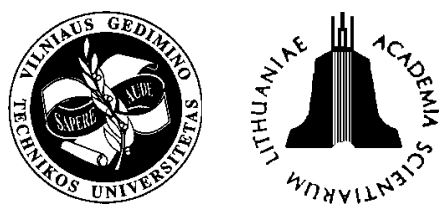

ISSN 1648-4142 TRANSPORT

http:/www.vtu.lt/english/editions

\title{
KNOWLEDGE BASED EXPERT SYSTEM APPROACH TO INSTRUMENTATION SELECTION (INSEL)
}

\author{
Sudhikumar Barai ${ }^{1}$, Padmesh Charan Pandey ${ }^{2}$ \\ ${ }^{1}$ Assistant Professor, Dept of Civil Engineering Indian Institute of Technology, \\ Kharagpur 721302 India.E-mail: skbarai@civil.iitkgp.ernet.in \\ 2 Professor, Dept of Civil Engineering Indian Institute of Science, \\ Bangalore 560012 India.E-mail:pcpandey@civil.iisc.ernet.in
}

Received 2004-02-02; accepted 2004-04-15

\begin{abstract}
The selection of appropriate instrumentation for any structural measurement of civil engineering structure is a complex task. Recent developments in Artificial Intelligence (AI) can help in an organized use of experiential knowledge available on instrumentation for laboratory and in-situ measurement. Usually, the instrumentation decision is based on the experience and judgment of experimentalists. The heuristic knowledge available for different types of measurement is domain dependent and the information is scattered in varied knowledge sources. The knowledge engineering techniques can help in capturing the experiential knowledge. This paper demonstrates a prototype knowledge based system for INstrument SELection (INSEL) assistant where the experiential knowledge for various structural domains can be captured and utilized for making instrumentation decision. In particular, this Knowledge Based Expert System (KBES) encodes the heuristics on measurement and demonstrates the instrument selection process with reference to steel bridges. INSEL runs on a microcomputer and uses an INSIGHT 2+ environment.
\end{abstract}

Keywords: artificial intelligence, bridge, instrumentation, knowledge based expert system, production rule, selection assistant.

\section{Introduction}

The damage assessment of a structure is based on its behavioral response reflected in forces and deformation resulting from different loading conditions. These behavioral responses are measured by instrumentation of the structure. The selection of suitable instrumentation is always a complex task especially for in-situ measurements of large civil engineering structures. Instruments with varieties of specifications are available in the market, which makes the instrumentation decision a difficult task indeed. Instrumenting a civil engineering structure is still a heuristic art and depends very much on the level of experience and expertise of the experimentalists. The recent developments in the computer science in general and Artificial Intelligence (AI) in particular can greatly help in organizing and utilizing the vast experiential knowledge available in the domain of instrumentation. Though the information about instrumentation can be stored using relational database approach, the retrieval of data is passive and hence, is not quite suitable for the instrumentation selection process. The problem of decision making with heuristics and quali- tative information can be efficiently dealt with the use of knowledge engineering techniques. The Knowledge Based Expert System (KBES) suitably designed can handle not only partial information, but also allow efficient user- interaction in the selection process. This paper presents and illustrates a knowledge based system for INstrumentation SELection (INSEL) developed using a commercial Expert System (ES) Shell in order to help in instrumentation decision in the context of in-situ measurements of civil engineering structures. A general architecture of INSEL is presented highlighting aspects of knowledge acquisition, knowledge representation and instrumentation decision. The knowledge-tree has been classified considering the types of structures, their materials of construction and their structural responses to be measured. In particular the knowledge-tree has been illustrated for instrumentation of steel bridge structures (Fig 1). Production rules have been used for knowledge representation in INSIGHT 2+ environment [1]. In the prototype expert system INSEL the selection of the instrumentation and decision on range of the instrument location are advocated basing on sensitivity study [2] along with the domain specific heuristics. 


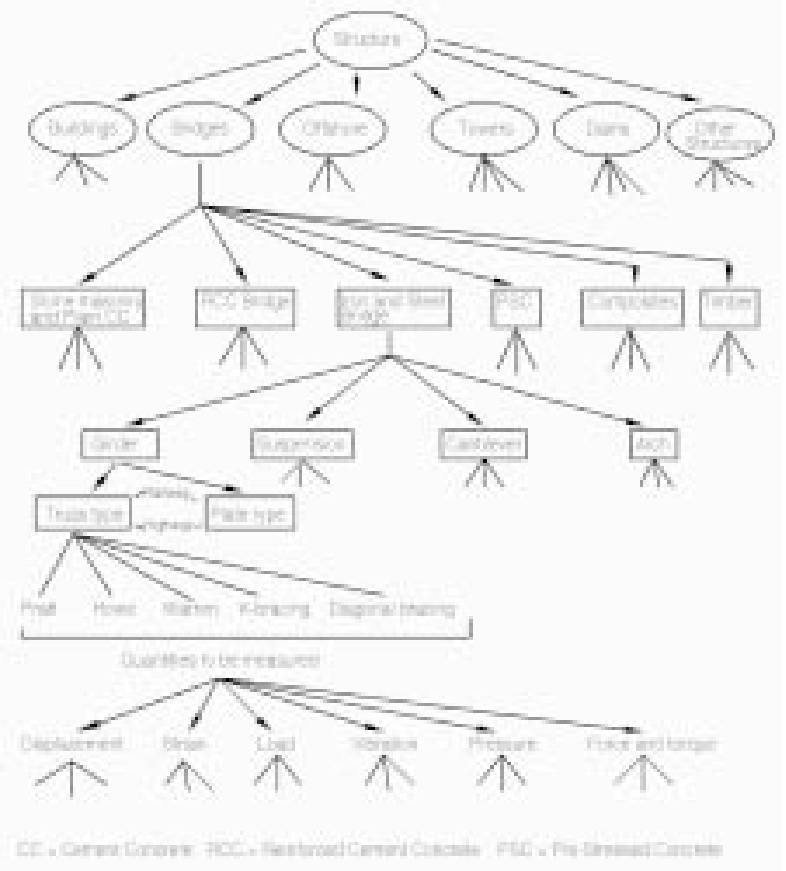

Fig 1. Knowledge-Tree

\section{Knowledge engineering: backgorund}

In "Knowledge Engineering", the term "engineering" implies the existence of some raw material which can indeed be engineered. Expert system offers the possibility to formulate a system to encode and utilize the knowledge of a domain expert which combines the background of many experts into a single, permanent decision support system for assessing the structural damage. If maintained over time, such a system could become a repository for corporate knowledge, so that experience need not to be lost when engineers retire or resign.

\subsection{Knowledge acquisition}

Acquisition of domain knowledge is a major problem in the development of an Expert System in the field of structural engineering. Though, expertise related to design has been largely codified, "Enquiry" is suggested as a way of elicitation of engineering knowledge necessary in the context of damage assessment. However, the task of acquiring engineering knowledge [3] in general may include the following aspects:

- Elicitation of distinction

- Decomposition of the main domain problem

- Combining of uncertain information

- Specification and/or choice of the most appropriate elicitation/acquisition technique

- Integration of different data/information types

- Use of multiple sources of knowledge

- Use of different sources of knowledge
- Organization and formulation of elicited knowledge(Knowledge representation)

- Feed-back between knowledge and domain engineer

- Verification of compiled/formatted knowledge

It is generally observed that the raw knowledge obtained from experts/sensors may contain some noise.

The knowledge sensors available for obtaining bridge inspection data can be identified as Visual, Photographic and Nondestructive Evaluation (NDE) instrumentation etc. However, the raw knowledge obtained from such sensors need to be examined by experts and processed for imprecision, uncertainty, vagueness and other forms of noise.

\subsection{Knowledge representation}

Knowledge has different forms. It can be certain or uncertain, formalized or unformalized, structured or unrelated, etc. It can be found in formulas, tables, statements, traditional practices or embeded in methodologies, but when it has to be translated in such a way that it can be stored and used by a computer, a knowledge representation mechanism has to be chosen. The issues that affect the selection of knowledge representation are naturalism of representation, efficiency of storage and manipulation, and consistency and compatibility with the rest of the representation in the system. There is no consensus how to classify the different classes of knowledge representation.

\section{Object Descriptors}

(i) Frames

A frame is a structure that represents a concept. It can have any number of attributes or properties attached to it; some of the properties can be relationship. An attribute may have any number of values. Other features of a frame are as follows $[4,5]$ :

- A frame contains information about a prototypical instance.

- Reasoning with frames is a process of matching prototypes against specific individuals.

- Frames are often represented as taxonomic hierarchies for efficient representation and reasoning.

- The information within a single frame is typically expressed as a collection of slots, each of which has one or more values in it.

- $\quad$ Frame concept is a specific case of another representation structure: semantic networks.

(ii) Objects, Parameter Values, Logics and Rules All entities except parts of message, comments, and certain punctuation symbols are objects. Usually 
Object Oriented Programming System (OOPS) utilizes objects that incorporate provisions for message passing between objects. Attached to these objects are procedures that can be activated by the receipt of messages. Parameter-value pairs can also represent declarative knowledge by the use of logic notation and to some extent, by rules [6].

\section{Actions}

They change a situation and/or modify the relevant database

\section{(i) Production Rules}

First generation expert systems are rule based systems. These are composed of rules, working memory and a rule interpreter. In their simplest form rules are condition-action pairs. These rules may be grouped together in modules for easy maintenance and rapid access. Some often praised attributes of rule based systems are modularity, modifiability and understandability. Each rule represents a chunk of knowledge. If rules are dependent, rules can be added to a knowledge base without change in the existing rules. Hayes-Roth et al. [7, 8] has given introductory document for a rule based system.

\section{(ii) Examples}

They indicate the conclusions and decisions reached. For the knowledge acquisition and from inductive systems point of view, examples are desirable, as they are much easier to elicit from experts than rules and also often in a natural form of domain knowledge.

\section{(iii) Predicate Logics}

Reasoning involves drawing conclusions from known knowledge. To automate reasoning one needs to have a language for representing knowledge and a mechanism for manipulating knowledge in order to draw a conclusion. One powerful way for representing and manipulating, and thus reasoning about, knowledge is predicate logic and theorem proving [9]. Theorems, axioms and any knowledge that can be formalized into a first order logic notation can naturally be mapped into logic-representation. One of the greatest advantages of representation using predicate logics is that syntactic inference is possible and is guaranteed to be valid. This characteristic makes it possible to develop automatic inference systems that are extremely powerful [10].

\section{(iv) Procedures}

Procedures are commonly used and known representation structures to engineers. Traditional numerical formulas map in a straightforward way into procedures. Recently, procedure based environments are available where symbolic data handling is possible. Sequential execution of structures, iteration and re- cursion are the three control schemes available to procedures. A procedure can be used as an action of a procedure rule, or as the mechanism that manipulates the information contained in a fact [6].

The knowledge acquisition, knowledge processing and knowledge representation are still an active area of AI research.

\section{Knowledge engineering aspects in instrumentation selection paradigm}

In the development of any Knowledge Based Expert Systems, as discussed earlier, basically two issues are very important namely Knowledge acquisition and Knowledge representation for problem domain. They are discussed below.

\subsection{Knowledge acquisition}

In this study the task of knowledge acquisition is approached by identifying the knowledge sources in the said domain [11-15].

\subsection{Knowledge representation}

Production rules are the most widely knowledge representation formalism in expert system available in ES shells. In this paper, for the selection of instrumentation production rules under INSIGHT $2+$ environment are adopted for knowledge representation.

\section{Expert system shell - insight 2+: general features}

INSIGHT $2+$, a commercially available expert system development tool runs on IBM PC (or compatible) microcomputers. It is easy to use and reasonably well documented. It allows easy integration with algorithmic program written in procedural languages. Also, its knowledge base can be compiled and is generally available. The other features of the INSIGHT $2+$ are as follows:

- INSIGHT 2+ uses the Production Rule Language (PRL). For the development of Knowledge Bases PRL provides the framework for organizing information and knowledge. The facilities include Confidence Factor and mathematical functions such as trigonometric and exponential.

- The inference engine employs both forward and backward chaining inference mechanisms.

- The user interface provides a user-friendly environment for two-way communication between the user and the knowledge base. This includes help facility, explanation facility (Why?, What is reasoning ?, etc.) through the "report" system.

- It handles mixed symbolic/ algorithmic processing well. 


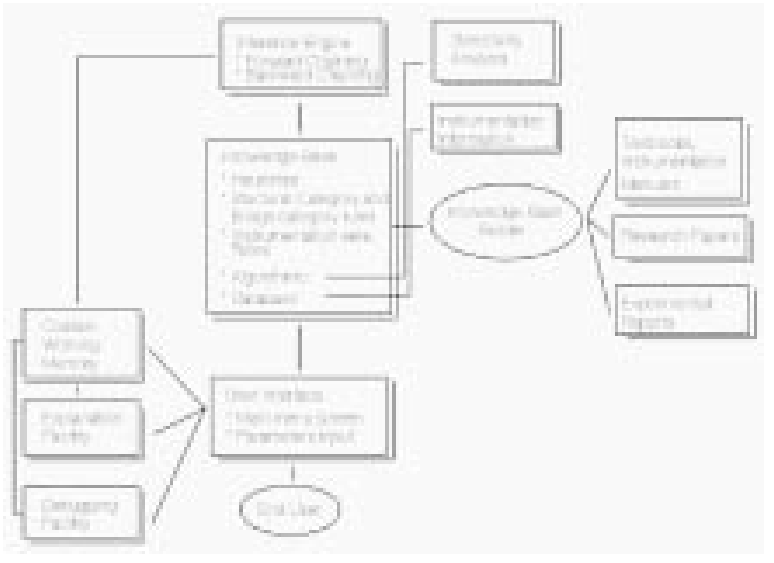

Fig 2. Knowledge Based System Architecture of INSEL

\section{Knowledge based system structure of insel}

The developed prototype expert system called INSEL is written using INSIGHT $2+$. The main components of INSEL consists of the inference engine, the user interface, the modular instrumentation knowledge base, database with instrumentation information. INSEL architecture is shown in Fig 2. The knowledge-tree has been shown in Fig 1.

The typical production rule of INSEL in INSIGHT 2+ environment is as follows:

RULE Instrument 1

IF Quantities to be measured IS Displacement

AND Railway truss IS Pratt type

AND Range_of_instrument ARE High

AND Economy_of_the_instrument ARE Not expensive

AND Output_size_of_instrument ARE High

AND Electronics_requirements ARE Minimum

THEN carry on

AND at least one succeeded

AND Instrument Category := "Instrument 1"

AND DISPLAY Instru_1

The knowledge base of INSEL is the depository of static, domain specific knowledge consisting of the facts and rules pertaining to structural instrumentation and testing. INSEL knowledge base has been structured in modules. It has:

- A set of knowledge modules, containing rules/ facts specific to the instrumentation applicable to civil engineering and

- A main module containing general rules/facts common to selection of any instrumentation as well as rules/facts specific to the determination of the appropriate INSEL (Fig 2).

An advantage of this modular arrangement is that the knowledge can be easily modified, updated and expanded.

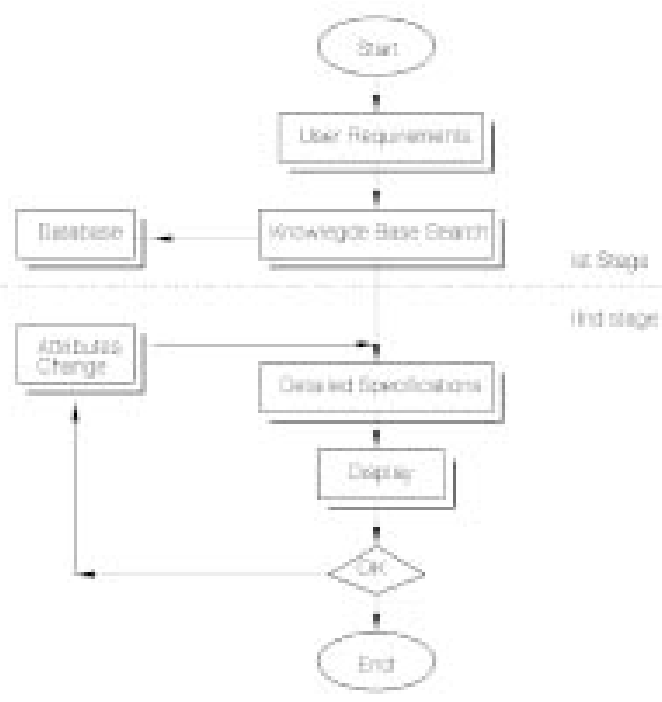

Fig 3. INSEL Operation

\section{Insel environment}

INSEL follows two processing steps which are shown in Fig 3.

In step I the user is guided through a series of pre-defined questions which are qualitative in nature in order to establish one or more rules supporting INSEL knowledge base that meet user requirements. The number and the sequence of the questions asked is dependent on the user input. In step II the user can interactively explore alternate instrumentation and select the instrumentation that best meets the overall requirements. A built-in cyclic control structure allows the user to re-specify previously indicated user requirements for generating new recommendations in case earlier ones are not acceptable.

\section{Consultation session with insel}

The scope of INSEL at present is limited to the instrumentation for displacement quantity with reference to steel bridges. The INSEL guides the user in an instrumentation selection and decision making process providing various menus and explanation. A typical consultation session with INSEL is given in Figs 4-12.

For the user input INSEL displays the instrumentation for user application with certain features (e.g. Range, temperature range, resolution, frequency response etc.) as shown in Fig 9. The recommended instrumentation, Linear Displacement Transducer (Transduction principle: Resistive wire-wound potentiometer) is the instrumentation with high range, economical, high output size and minimum electronics requirements (Fig 9). This instrumentation satisfies the user requirements specified up to that stage of consultation. 


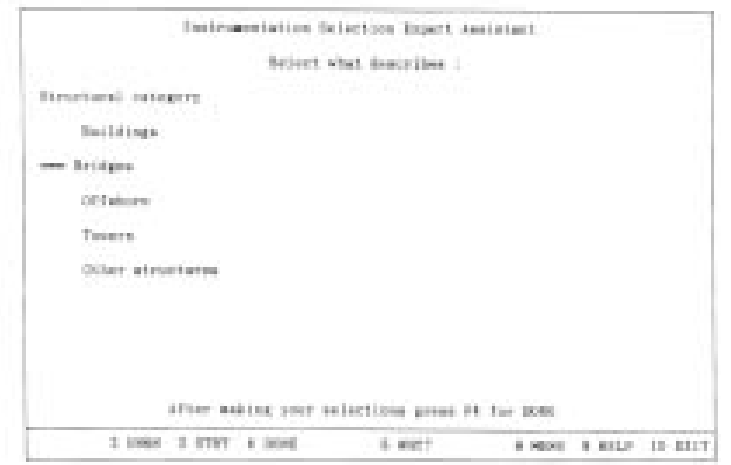

Fig 4. Typical user input for structural category

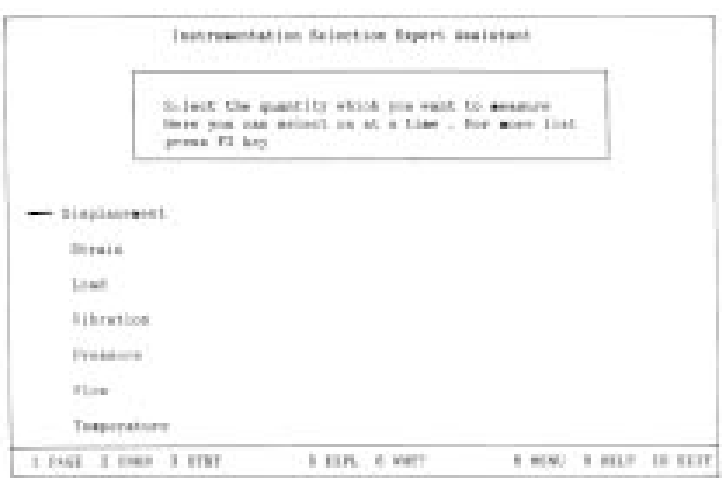

Fig 5. Typical user input for girder type of steel bridge structure

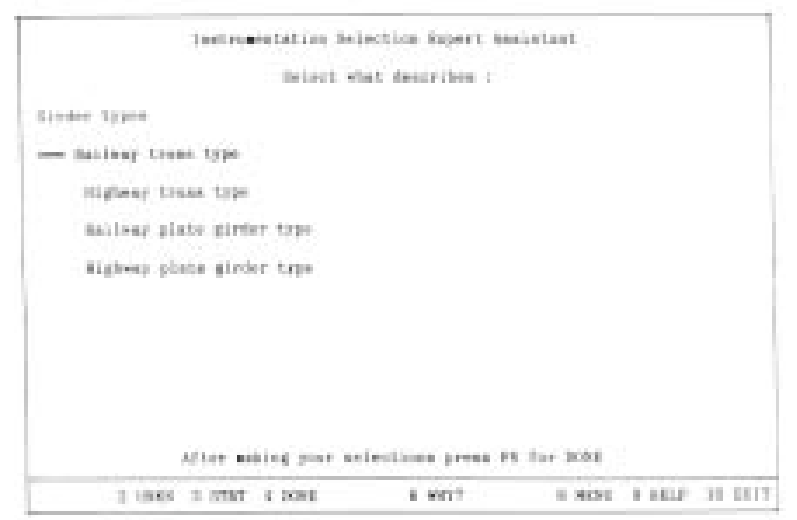

Fig 6. Typical user input for quantity to be measured

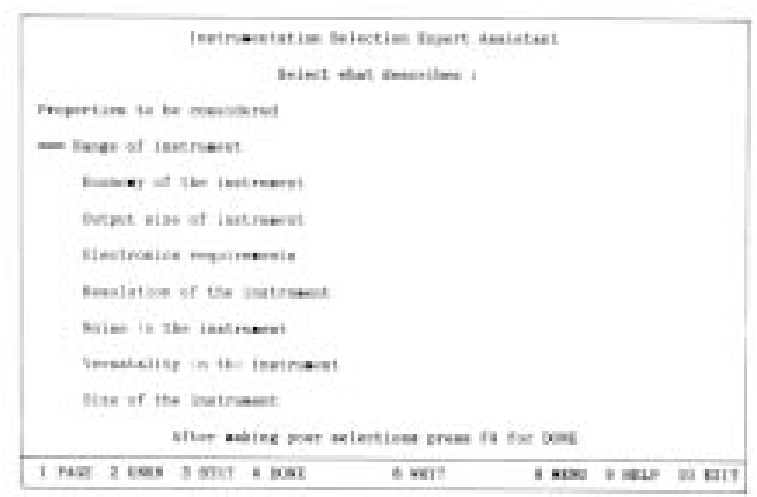

Fig 7. Input queries for properties of instrumentation

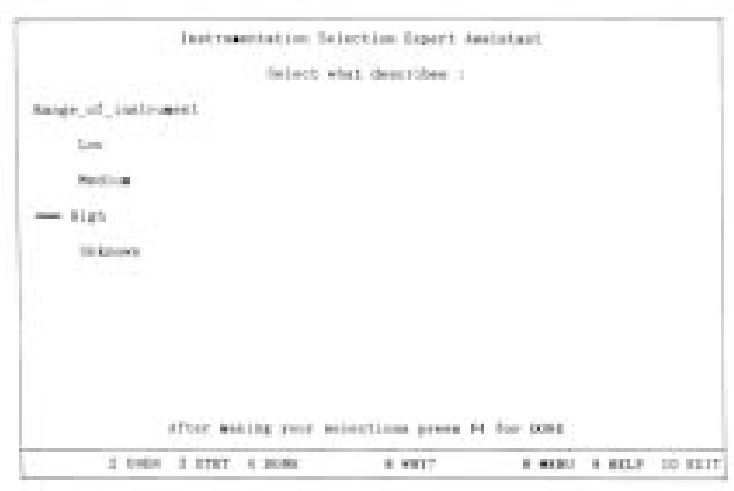

Fig 8. Typical user input for range of instrument

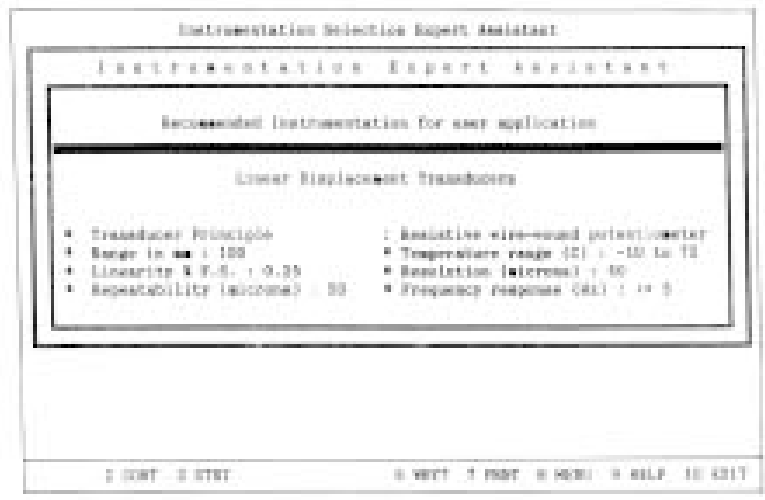

Fig 9. INSEL recommendation for Instrumentation

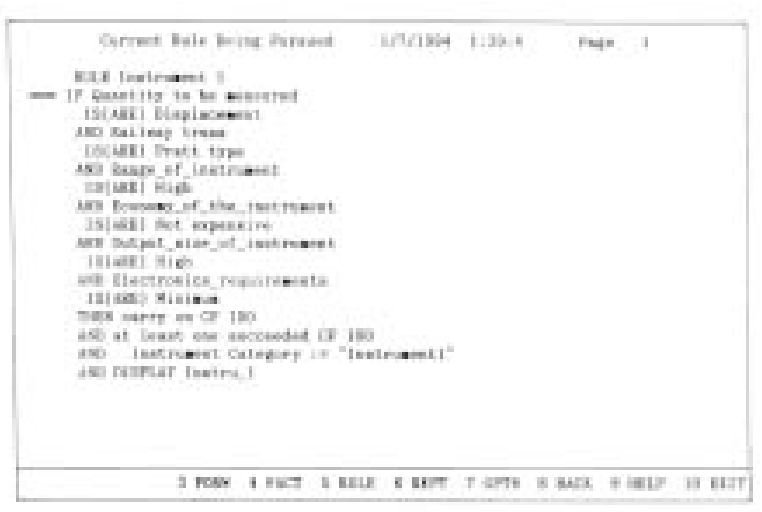

Fig 10. Typical rule display

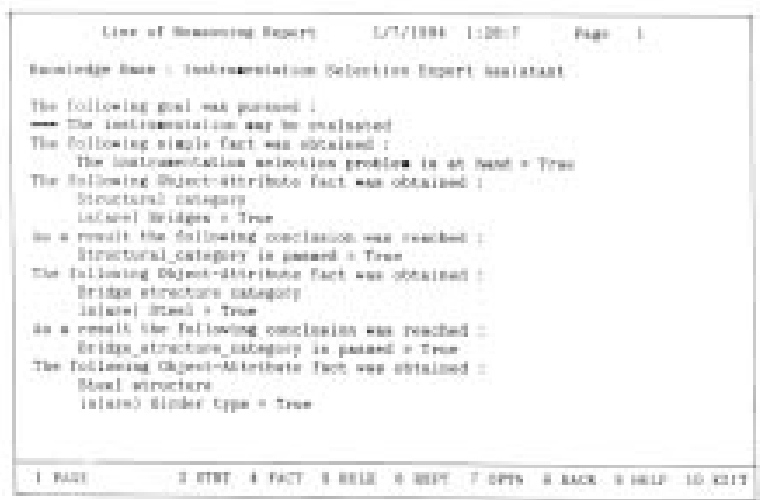

Fig 11. Example of Report system for line of reasoning 


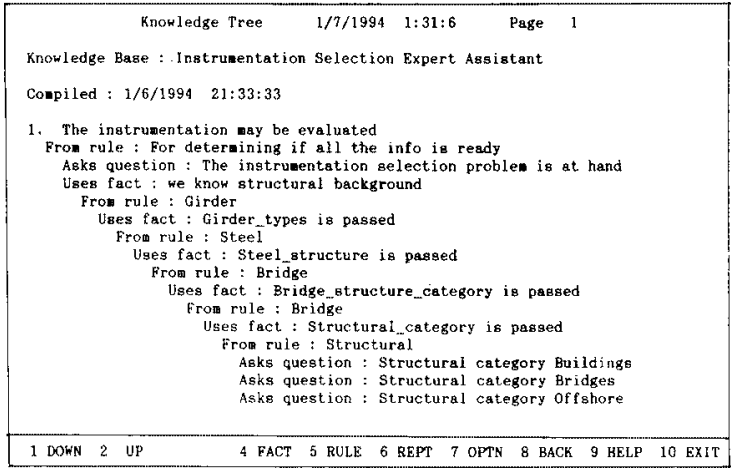

Fig 12. Typical Knowledge-tree display

\section{Future projections of INSEL}

For the current version of INSEL the following developments are possible:

- For the fast information retrieval about the instrumentation the database can be developed in database -III and can be linked in INSIGHT $2+$ environment

- INSEL can be enhanced for the other types of structures using the same knowledge structure.

- INSEL provides advice on instrumentation for displacement, but the same can be extended for strain, vibration, load etc.

\section{Conclusions}

The paper presented a knowledge-based approach for the instrumentation selection of civil engineering structures. A demonstration prototype KBES for instrumentation selection INSEL has been implemented under INSIGHT 2+ environment. It uses a modularized knowledge base for fast, efficient search as well as data retrieval. INSEL provides a knowledge base structure for convenient and systematic compilation and inference using the available Instrumentation information. Currently the knowledge base of INSEL contains limited domain specific knowledge with reference to steel bridges. However, it can be easily extended to other type of structures. Issues related to the degree of accuracy of measurement, economic considerations and alternative selection strategy with fuzzy ranking could also be incorporated.

\section{References}

1. INSIGHT $2+$, Reference Manual, Version 1.0, Level 5 Research Inc., Indialantic, Florida, 1986.

2. Pandey, P. C. and Barai, S. V., (1993). Sensitivity based Instrumentation for structural measurements, Journal of Instrumentation Society of India, 24(3-4), 101111

3. Jovanovic, A. S.; Kussmaul, K. F.; Lucia, A. C., and Bonissone, P. P. (Eds.) Expert systems in structural safety assessment, Lecture notes in engineering: 53, Springer Verlag, 1989.

4. Fikes, R. and Kehler, T. (1985). The role of framebased representation in reasoning, Communications of ACM, 28(9), p. 904-920.

5. Sarma, V. V. S.; Vishwanadham, N.; Yagnanarayan, B. and Deekshatulu, D. L. Artificial intelligence and expert systems technologies in Indian context, Vol 1 and 2, Tata McGraw Hill Publishing Company Ltd., 1991.

6. Patterson, D. W., Introduction to artificial intelligence and expert systems, Prentice Hall, Englewood Cliffs, New Jersey, 1990.

7. Hayes-Roth, F.; Waterman, D. A., and Lenat, D. (Eds.), Building Expert Systems, Addison-Wesley, 1983.

8. Rolston, D. W. Principles of Artificial Intelligence and Expert System Development, McGraw Hill Book Company, 1988.

9. Chang, C. L. and Lee, R. C. Symbolic logic and mathematical theorem proving, Academic Press, New York, 1973.

10. Rich, E. Artificial intelligence, McGraw Hill Book Company, 1983.

11. Garas, F. K.; Clarke, J. L.; Armer, G. S. T. Structural assessment: The use of full and large scale testing, Butterworth, 1987.

12. Nowak, A. S. Bridge Evaluation, Repair and Rehabilitation, Kluwer Academic Publication, 1990.

13. Rangan, C. S.; Sarma, G. R. and Mani, V. S. V. Instrumentation Devices and Systems, Tata McGraw-Hill Publishing Company Limited, 1989.

14. Sabnis, G. M.; Harris, H. G.; White, R. N. and Mirza, M. S. Structural modelling and experimental techniques, Prentice-Hall, Englewood Cliffs, 1983.

15. Brylawski, E. D. (1986). Going afield with instrumentation, ASCE Civil Engineering, Aug., 49-51. 